\title{
Axiomatic properties of inconsistency indices for pairwise comparisons
}

\author{
Matteo Brunelli \\ Systems Analysis Laboratory, Department of Mathematics and Systems Analysis \\ Aalto University, P.O. box 11100, FIN-00076 Aalto, Finland \\ e-mail: matteo.brunelli@aalto.fi \\ Michele Fedrizzi \\ Department of Industrial Engineering \\ University of Trento, Via Mesiano 77, I-38123 Trento, Italy \\ e-mail: michele.fedrizzi@unitn.it
}

\begin{abstract}
Pairwise comparisons are a well-known method for the representation of the subjective preferences of a decision maker. Evaluating their inconsistency has been a widely studied and discussed topic and several indices have been proposed in the literature to perform this task. Since an acceptable level of consistency is closely related with the reliability of preferences, a suitable choice of an inconsistency index is a crucial phase in decision making processes. The use of different methods for measuring consistency must be carefully evaluated, as it can affect the decision outcome in practical applications. In this paper, we present five axioms aimed at characterizing inconsistency indices. In addition, we prove that some of the indices proposed in the literature satisfy these axioms, while others do not, and therefore, in our view, they may fail to correctly evaluate inconsistency.
\end{abstract}

Keywords: Pairwise comparisons, inconsistency indices, axiomatic properties, analytic hierarchy process.

\section{Introduction}

Pairwise comparisons have been used in some operations research methods to represent the preferences of experts and decision makers over sets of alternatives, criteria, features and so on. For simplicity, in this paper we shall speak of alternatives only, bearing in mind that it is a reductive view. The main advantage in using pairwise comparisons is that they allow the decision maker to compare two alternatives at a time, thus reducing the complexity of a decision making problem, especially when the set under consideration is large, and serve as a starting point to derive a priority vector which is the final rating of the alternatives. Pairwise comparisons have been used in well-known decision analysis methods as, for instance, the Analytic Hierarchy 
Process (AHP) by Saaty (1977) (see Ishizaka and Labib (2011) for an updated discussion), and its generalizations, which have been proved effective in solving many decision problems (Ishizaka et al., 2011).

In the literature, and in practice, it is assumed that the dependability of the decision is related to the consistency of his/her pairwise judgments. That is, the more rational the judgments are, the more likely it is that the decision maker is a good expert with a deep insight into the problem and pays due attention in eliciting his/her preferences. Similarly, if judgments are very intransitive and irrational, it is more plausible that the expert expressed them with scarce competence, since he/she would lack the ability to rationally discriminate between different alternatives. This is summarized by Irwin's thesis claiming that “...preference is exactly as fundamental as discrimination and that if the organism exhibits a discrimination, it must also exhibit a preference and conversely" (Irwin, 1958). Following Saaty (1994) the approach to decision making based on pairwise comparisons, and the AHP in particular, is grounded in the relative measurement theory and it is in this framework that Saaty (1993) too claimed that pairwise comparisons should be 'near consistent' to ensure that they are a sufficiently good approximation of the decision makers' real preferences. This seems to support the importance of having reliable tools capable of capturing the degree of inconsistency of pairwise comparisons. The importance of having reliable inconsistency indices becomes even more evident when one considers that their practical use has gone beyond the sole quantification of inconsistency. For instance, they have been employed by Lamata and Peláez (2002) and Shiraishi et al. (1999) to estimate missing comparisons, by Harker (1987) to derive ratings of alternatives from incomplete preferences and by $\mathrm{Xu}$ and Cuiping (1999) and Xu and Xia (2013) to improve the consistency of pairwise comparisons.

On this fertile ground, researchers have proposed various inconsistency indices-functions associating pairwise comparisons to real numbers representing the degrees of inconsistency of the pairwise judgments. In this paper we concern ourselves with the fact that inconsistency indices have been introduced heuristically and independently from each other, neither referring to a general definition, nor to a set of axiomatic properties. Hence, this paper introduces some axiomatic properties for inconsistency indices and shows that some indices proposed in literature fail to satisfy these axioms. This paper is outlined as follows. In Section 2 we introduce preliminary notions and the notation. In Section 3 we shortly define the inconsistency indices that are studied in this paper. Next, in Section 4 we introduce and interpret five axioms and in Section 5 we present results regarding the inconsistency indices and prove that some of them satisfy the required axioms while four others do not. For a simpler description, some proofs are given in the appendix. In Section 6, we conclude the discussion of the axioms and draw the conclusions.

Throughout this paper, we refer to 'inconsistency indices', since what they really measure is the amount of inconsistency in pairwise comparisons. Nevertheless, in literature such indices are often referred to as 'consistency indices', while both expressions refer to an index which estimates the deviation from consistency.

\section{Preliminaries}

Pairwise comparison matrices are convenient tools to model the decision makers' pairwise intensities of preference over sets of alternatives. Formally, given a set of alternatives $X=$ 
$\left\{x_{1}, \ldots, x_{n}\right\}(n \geq 2)$, Saaty (1977) defined a pairwise comparison matrix $\mathbf{A}=\left(a_{i j}\right)_{n \times n}$ as a positive and reciprocal square matrix of order $n$, i.e. $a_{i j}>0, a_{i j} a_{j i}=1, \forall i, j$, where $a_{i j}$ is an estimation of the degree of preference of $x_{i}$ over $x_{j}$. A pairwise comparison matrix is consistent if and only if the following transitivity condition holds:

$$
a_{i k}=a_{i j} a_{j k} \forall i, j, k
$$

Property (11) means that preferences are fully coherent, and each direct comparison $a_{i k}$ between $x_{i}$ and $x_{k}$ is confirmed by all indirect comparisons $a_{i j} a_{j k} \forall j$. If and only if $\mathbf{A}$ is consistent, then there exists a priority (or weight) vector $\mathbf{w}=\left(w_{1}, \ldots, w_{n}\right)$ such that

$$
a_{i j}=\frac{w_{i}}{w_{j}} \forall i, j .
$$

Crawford and Williams (1985) proved that if $\mathbf{A}$ is consistent, then the components of vector $\mathbf{w}$ can be obtained by using the geometric mean method,

$$
w_{i}=\left(\prod_{j=1}^{n} a_{i j}\right)^{\frac{1}{n}} \forall i
$$

Another method for obtaining the priorities is the eigenvector method by Saaty (1977). Namely, the priority vector $\mathbf{w}$ is the solution of the following equation

$$
\mathbf{A} \mathbf{w}=\lambda_{\max } \mathbf{w}
$$

where $\lambda_{\max }$ is the maximum eigenvalue of $\mathbf{A}$ whose existence and properties refer to the PerronFrobenius theorem. If $\mathbf{A}$ is consistent, both methods yield the same priority vector, while they may give different vectors if $\mathbf{A}$ is not consistent.

We define the set of all pairwise comparison matrices as

$$
\mathcal{A}=\left\{\mathbf{A}=\left(a_{i j}\right)_{n \times n} \mid a_{i j}>0, a_{i j} a_{j i}=1 \forall i, j, n>2\right\} .
$$

Similarly, the set of consistent pairwise comparison matrices $\mathcal{A}^{*} \subset \mathcal{A}$ is defined as

$$
\mathcal{A}^{*}=\left\{\mathbf{A}=\left(a_{i j}\right)_{n \times n} \mid \mathbf{A} \in \mathcal{A}, a_{i k}=a_{i j} a_{j k} \forall i, j, k\right\} .
$$

Seen from this perspective, a matrix can either be consistent or non-consistent (inconsistent). However, often, degrees of inconsistency are assigned to pairwise comparison matrices so that, if the inconsistency is not too high, the judgments in the pairwise comparison matrix are taken to be as sufficiently reliable. To sum up, the idea is that a good inconsistency index should indicate 'how much' the pairwise comparison matrix deviates from the full consistency. Thus, an inconsistency index $I$ is a real-valued function

$$
I: \mathcal{A} \rightarrow \mathbb{R}
$$

Although the codomain of the definition is the set of real numbers, each inconsistency index $I$ is univocally associated to a given image $\operatorname{Im}(I) \subseteq \mathbb{R}$. 


\section{Inconsistency indices}

In this section we shortly recall some inconsistency indices, giving a special emphasis to those which will be analyzed in the next section with respect to the five axioms. For a survey the reader can refer to Brunelli et al. (2013a). The first index is the Consistency Index, proposed by Saaty (1977).

Definition 1 (Consistency Index (Saaty, 1977)). Given a pairwise comparison matrix $\mathbf{A}$, the Consistency Index is defined as

$$
C I(\mathbf{A})=\frac{\lambda_{\max }-n}{n-1}
$$

where $\lambda_{\max }$ is the principal right eigenvalue of $\mathbf{A}$.

Formula (6) refers to the property that the maximum eigenvalue $\lambda_{\max }$ of a pairwise comparison matrix $\mathbf{A}$ is equal to $n$ if and only if the matrix is consistent, and greater than $n$ otherwise. Saaty proposed also a more suitable measure of inconsistency, called Consistency Ratio (CR),

$$
C R(\mathbf{A})=\frac{C I(\mathbf{A})}{R I}
$$

where $R I$, Random Index, is a suitable normalization factor.

Golden and Wang (1989) proposed a method to compute the deviations between the entries of a pairwise comparison matrix and their theoretical values $w_{i} / w_{j}$.

Definition 2 (Index $G W$ (Golden and Wang, 1989)). Given a pairwise comparison matrix $\mathbf{A}=$ $\left(a_{i j}\right)_{n \times n} \in \mathcal{A}$, the entries of every column are normalized by dividing them by the sum of the elements of their column $\sum_{i=1}^{n} a_{i j}$. Let us denote by $\overline{\mathbf{A}}=\left(\bar{a}_{i j}\right)_{n \times n}$ the new normalized matrix. Each priority vector associated (by either (3) or (4)) with $\mathbf{A}$ is normalized by dividing each component by the sum of the components and denoted by $\overline{\mathbf{w}}=\left(\bar{w}_{1}, \ldots, \bar{w}_{n}\right)$, so that $\sum_{i=1}^{n} \bar{w}_{i}=1$. The inconsistency index $G W$ is defined as

$$
G W(\mathbf{A})=\frac{1}{n} \sum_{i=1}^{n} \sum_{j=1}^{n}\left|\bar{a}_{i j}-\bar{w}_{i}\right| .
$$

Crawford and Williams (1985), and later Aguaròn and Moreno-Jimènez (2003), proposed and refined an index that also computes distances between the decision maker's judgments and their theoretical values obtained as ratios $w_{i} / w_{j}$.

Definition 3 (Geometric Consistency Index (Aguaròn and Moreno-Jimènez, 2003)). Given a pairwise comparison matrix of order $n$, the Geometric Consistency Index GCI is defined as follows

$$
G C I(\mathbf{A})=\frac{2}{(n-1)(n-2)} \sum_{i=1}^{n-1} \sum_{j=i+1}^{n} \ln ^{2}\left(a_{i j} \frac{w_{j}}{w_{i}}\right),
$$

where the weights are obtained by means of the geometric mean method (3).

Barzilai (1998) formulated a normalized index based on squared errors. By using open unbounded scales, he stated several relevant algebraic and geometric properties. 
Definition 4 (Relative Error (Barzilai, 1998)). Given a pairwise comparison matrix $\mathbf{A} \in \mathcal{A}$, the relative error, $R E$, is defined as

$$
R E(\mathbf{A})=1-\frac{\sum_{i=1}^{n} \sum_{j=1}^{n}\left(\frac{1}{n} \sum_{k=1}^{n} \log a_{i k}-\frac{1}{n} \sum_{k=1}^{n} \log a_{j k}\right)^{2}}{\sum_{i=1}^{n} \sum_{j=1}^{n}\left(\log a_{i j}\right)^{2}} .
$$

for all matrices $\mathbf{A} \neq(1)_{n \times n}$, and zero if $\mathbf{A}=(1)_{n \times n}$.

Peláez and Lamata (2003) defined an inconsistency index for a pairwise comparison matrix as the average of all the determinants of its $3 \times 3$ submatrices, each containing a different transitivity of the original matrix.

Definition 5 (Index $C I^{*}$ (Peláez and Lamata, 2003)). Given a pairwise comparison matrix of order $n$, the index $C I^{*}$ is

$$
C I^{*}(\mathbf{A})=\sum_{i=1}^{n-2} \sum_{j=i+1}^{n-1} \sum_{k=j+1}^{n}\left(\frac{a_{i k}}{a_{i j} a_{j k}}+\frac{a_{i j} a_{j k}}{a_{i k}}-2\right) /\left(\begin{array}{l}
n \\
3
\end{array}\right) .
$$

Shiraishi et al. (1998) proposed the coefficient $c_{3}$ of the characteristic polynomial of $\mathbf{A}$ as an index of inconsistency. Brunelli et al. (2013b) proved that index $C I^{*}$ is proportional to $c_{3}$.

Stein and Mizzi (2007) considered the general result that the columns of a consistent pairwise comparison matrix are proportional, i.e. $\operatorname{rank}(\mathbf{A})=1$, if and only if $\mathbf{A}$ is consistent. Thus, they formulated an index which takes into account how far the columns are from being proportional to each other.

Definition 6 (Harmonic Consistency Index (Stein and Mizzi, 2007)). Let A be a pairwise comparison matrix and $s_{j}=\sum_{i=1}^{n} a_{i j}$ for $j=1, \ldots, n$. Then, the harmonic consistency index is

$$
H C I(\mathbf{A})=\frac{(H M(\mathbf{A})-n)(n+1)}{n(n-1)},
$$

where $H M(\mathbf{A})$ is the harmonic mean of $\left(s_{1}, \ldots, s_{n}\right)$ :

$$
H M(\mathbf{A})=\frac{n}{\sum_{j=1}^{n} \frac{1}{s_{j}}}
$$

Koczkodaj (1993) and Duszak and Koczkodaj (1994) introduced a max-min based inconsistency index which was later compared with CI by Bozóki S. and Rapcsák (2008). Cavallo and D'Apuzzo (2009) characterized pairwise comparison matrices by means of Abelian linearly ordered groups and stated their inconsistency index in this general framework.

Another index, $N I_{n}^{\sigma}$, was introduced by Ramík and Korviny (2010) to estimate the inconsistency of pairwise comparison matrices with elements expressed as triangular fuzzy numbers. Expressing judgments in such a way is popular to account for uncertainties in the decision making process. Nevertheless, pairwise comparison matrices can be seen as special cases of matrices with fuzzy entries and therefore this index can be introduced in the context of pairwise comparison matrices with real entries. 
Definition 7 (Index $N I_{n}^{\sigma}$ (Ramík and Korviny, 2010)). Given a real number $\sigma>0$ and a pairwise comparison matrix $\mathbf{A} \in \mathcal{A}$ of order $n$ with entries in the interval $[1 / \sigma, \sigma]$, the index $N I_{n}^{\sigma}$ is defined as

$$
N I_{n}^{\sigma}(\mathbf{A})=\gamma_{n}^{\sigma} \max _{i, j}\left\{\left|\frac{w_{i}}{w_{j}}-a_{i j}\right|\right\}
$$

where the weights are obtained by means of the geometric mean method (3) and

$$
\gamma_{n}^{\sigma}= \begin{cases}\frac{1}{\max \left\{\sigma-\sigma^{\frac{2-2 n}{n}}, \sigma^{2}\left(\left(\frac{2}{n}\right)^{\frac{2}{n-2}}-\left(\frac{2}{n}\right)^{\frac{n}{n-2}}\right)\right\}}, & \text { if } \sigma<\left(\frac{n}{2}\right)^{\frac{n}{n-2}} \\ \frac{1}{\max \left\{\sigma-\sigma^{\frac{2-2 n}{n}}, \sigma^{\frac{2 n-2}{n}}-\sigma\right\}}, & \text { if } \sigma \geq\left(\frac{n}{2}\right)^{\frac{n}{n-2}}\end{cases}
$$

is a positive normalization factor.

Other notable indices are the parametric method by Osei-Bryson (2006) and the ambiguity index by Salo (1993).

\section{Axioms}

In spite of the large number of indices, the question on how well they estimate inconsistency of pairwise comparisons has been left unanswered. To answer this question, in this section we introduce and justify five properties to narrow the general definition of inconsistency index given in (5) and to shed light on those indices which do not satisfy minimal reasonable requirements. Throughout this and the next sections we are going to propose some examples in order to provide numerical and visual evidence of the necessity of the following axiomatic system.

\section{Axiom 1: Existence of a unique element representing consistency}

With axiom 1 (A1) we require that all the consistent matrices are identified by a unique real value of an inconsistency index. This allows to distinguish between matrices that either belong or do not to $\mathcal{A}^{*}$. Formally, $\mathrm{A} 1$ is as follows.

Axiom 1. An inconsistency index I satisfies A1, if and only if

$$
\exists ! \nu \in \mathbb{R} \text { such that } I(\mathbf{A})=\nu \Leftrightarrow \mathbf{A} \in \mathcal{A}^{*}
$$

Example 1. The following inconsistency index satisfies A1 with $\nu=0$.

$$
I(\mathbf{A})=\sum_{i=1}^{n} \sum_{j=1}^{n} \sum_{k=1}^{n}\left|a_{i k}-a_{i j} a_{j k}\right| .
$$

For sake of simplicity, we assume, without loss of generality, that, for every inconsistency index $I(\mathbf{A})$, the value $\nu$ associated with each consistent matrix is the minimum value of the index: $I(\mathbf{A}) \geq \nu \forall \mathbf{A} \in \mathcal{A}$. The assumption is that the more inconsistent is $\mathbf{A}$, the greater is $I(\mathbf{A})$. Some already introduced indices assume the opposite. By considering, for example, the index introduced by Shiraishi et al. (1998), it is $c_{3}(\mathbf{A}) \leq 0 \forall \mathbf{A} \in \mathcal{A}$, while the consistency value is $c_{3}(\mathbf{A})=\nu=0 \forall \mathbf{A} \in \mathcal{A}^{*}$. Nevertheless, in such cases it is sufficient to change the sign of the index to fulfill our assumption. 


\section{Axiom 2: Invariance under permutation of alternatives}

It is desirable that an inconsistency index does not depend on the order in which the alternatives are associated with rows and columns of $\mathbf{A}$. Therefore, an inconsistency index should be invariant under row-column permutations. To formalize this second axiom (A2), we recall that a permutation matrix is a square binary matrix $\mathbf{P}$ that has exactly one entry equal to 1 on each row and each column and 0's elsewhere (see Horn and Johnson 1985). We also recall that $\mathbf{P A P}^{\mathrm{T}}$ is the matrix obtained from $\mathbf{A}$ through the row-column permutations associated with $\mathbf{P}$.

Axiom 2. An inconsistency index I satisfies A2, if and only if

$$
I\left(\mathbf{P A P}^{\mathrm{T}}\right)=I(\mathbf{A}) \forall \mathbf{A} \in \mathcal{A}
$$

and for any permutation matrix $\mathbf{P}$.

Example 2. Given a pairwise comparison matrix $\mathbf{A}$ and a permutation matrix $\mathbf{P}$

$$
\mathbf{A}=\left(\begin{array}{ccc}
1 & 2 & 5 \\
1 / 2 & 1 & 2 \\
1 / 5 & 1 / 2 & 1
\end{array}\right) \quad \mathbf{P}=\left(\begin{array}{ccc}
0 & 1 & 0 \\
1 & 0 & 0 \\
0 & 0 & 1
\end{array}\right)
$$

one obtains

$$
\mathbf{P A P}^{\mathrm{T}}=\left(\begin{array}{lll}
0 & 1 & 0 \\
1 & 0 & 0 \\
0 & 0 & 1
\end{array}\right)\left(\begin{array}{ccc}
1 & 2 & 5 \\
1 / 2 & 1 & 2 \\
1 / 5 & 1 / 2 & 1
\end{array}\right)\left(\begin{array}{lll}
0 & 1 & 0 \\
1 & 0 & 0 \\
0 & 0 & 1
\end{array}\right)=\left(\begin{array}{ccc}
1 & 1 / 2 & 2 \\
2 & 1 & 5 \\
1 / 2 & 1 / 5 & 1
\end{array}\right)
$$

for which (15) is required to hold.

\section{Axiom 3: Monotonicity under reciprocity-preserving mapping}

Unlike the previous axioms, which were simple regularity conditions imposed to $I(\mathbf{A})$, axiom 3 (A3) is more constraining. The idea is that, if preferences are intensified, then an inconsistency index cannot return a lower value. However, before we formalize it, we describe its meaning. If all the expressed preferences indicate indifference between alternatives, it is $a_{i j}=1 \forall i, j$, and $\mathbf{A}$ is consistent. Going farther from this uniformity means having stronger judgments and this should not make their possible inconsistency less evident. In other words, intensifying the preferences (pushing them away from indifference) should not de-emphasize the characteristics of these preferences and their possible contradictions. Clearly, the crucial point is to find a transformation which can intensify preferences and preserve their structure at the same time. In the following, we are going to prove that such a transformation exists and is unique. Given $\mathbf{A}=\left(a_{i j}\right) \in \mathcal{A}$, we denote such transformation with $\hat{a}_{i j}=f\left(a_{i j}\right)$. The newly constructed matrix $\hat{\mathbf{A}}=\left(\hat{a}_{i j}\right)$ obtained from $\mathbf{A}$ by means of $f$ must be positive and reciprocal so that it still belongs to $\mathcal{A}$. Hence

$$
\hat{a}_{j i}=1 / \hat{a}_{i j}
$$

which is

$$
\begin{aligned}
f\left(a_{j i}\right) & =1 / f\left(a_{i j}\right) \\
f\left(1 / a_{i j}\right) & =1 / f\left(a_{i j}\right) \\
f\left(a_{i j}\right) f\left(1 / a_{i j}\right) & =1
\end{aligned}
$$


or, more compactly, with $a_{i j}=x$,

$$
f(x) f(1 / x)=1 .
$$

Equation (16) is a special case, for $y=1 / x$, of the well-known Cauchy functional equation

$$
f(x) f(y)=f(x y) .
$$

In fact, by substituting $x=1$ into (16), it is $f(1) f(1)=1$. Since $f$ must be positive, it follows $f(1)=1$. Then, (16) can also be written in the form $f(x) f(1 / x)=f(x(1 / x))=f(1)$. Taking into account that $x=a_{i j}>0$, it is therefore sufficient to assume the continuity of $f$ in order to obtain a unique non-trivial solution of (16) (see Aczel 1966)

$$
f(x)=x^{b}, \quad b \in \mathbb{R} .
$$

Therefore, the only continuous transformation $f\left(a_{i j}\right)$ preserving reciprocity is (18), i.e. $f\left(a_{i j}\right)=$ $a_{i j}^{b}$. In the following, we will denote matrix $\left(a_{i j}^{b}\right)_{n \times n}$ as $\mathbf{A}(b)$. Clearly, for $b>1$ each entry $a_{i j} \neq 1$ is moved farther from indifference value 1 , which represents an intensification of preferences:

$$
\begin{aligned}
b>1, \quad a_{i j}>1 & \Rightarrow a_{i j}^{b}>a_{i j}>1 \\
b>1, \quad 0<a_{i j}<1 & \Rightarrow 0<a_{i j}^{b}<a_{i j}<1 .
\end{aligned}
$$

The opposite occurs for $0<b<1$, thus representing a weakening of the preferences. For $b=0$ full indifference is obtained, $a_{i j}^{b}=1$, while $b<0$ corresponds to preference reversal. Moreover, transformation (18) is consistency-preserving, i.e. if $\mathbf{A}=\left(a_{i j}\right)$ is consistent, then also $\mathbf{A}(b)=\left(a_{i j}^{b}\right)$ is consistent. The proof is straightforward, since from $a_{i j} a_{j k}=a_{i k}$ immediately follows $a_{i j}^{b} a_{j k}^{b}=a_{i k}^{b}$. Furthermore, (18) is also the unique consistency-preserving transformation, the proof being similar to the one described above for reciprocity.

To summarize, the only continuous transformation that intensifies preferences and preserves reciprocity (and consistency) is $f\left(a_{i j}\right)=a_{i j}^{b}$ with $b>1$ and then A3 can be formalized.

Axiom 3. Define $\mathbf{A}(b)=\left(a_{i j}^{b}\right)_{n \times n}$. Then, an inconsistency index I satisfies A3 if and only if

$$
I(\mathbf{A}(b)) \geq I(\mathbf{A}) \quad \forall b>1, \quad \forall \mathbf{A} \in \mathcal{A} .
$$

Example 3. Consider the following matrix

$$
\mathbf{A}=\left(\begin{array}{ccc}
1 & 2 & 1 / 2 \\
1 / 2 & 1 & 2 \\
2 & 1 / 2 & 1
\end{array}\right)
$$

Then, modifying entries of $\mathbf{A}$ by means of function $f$ with exponent $b=3$ one obtains the following matrix

$$
\mathbf{A}(3)=\left(\begin{array}{ccc}
1^{3} & 2^{3} & 1 / 2^{3} \\
1 / 2^{3} & 1^{3} & 2^{3} \\
2^{3} & 1 / 2^{3} & 1^{3}
\end{array}\right)=\left(\begin{array}{ccc}
1 & 8 & 1 / 8 \\
1 / 8 & 1 & 8 \\
8 & 1 / 8 & 1
\end{array}\right)
$$

If an inconsistency index $I$ satisfies A3, then it must be $I(\mathbf{A}(3)) \geq I(\mathbf{A})$. In words, if A3 holds, then $\mathbf{A}(3)$ cannot be judged less inconsistent than $\mathbf{A}$.

Note that transformation $f\left(a_{i j}\right)=a_{i j}^{b}$ has been used for other scopes. Saaty (1977) himself proposed it in his seminal paper to show that his results on consistency were general enough to cover scales other than $[1 / 9,9]$. Such a function was also employed by Herrera-Viedma et al. (2004) to find a suitable mapping to rescale the entries of a pairwise comparison matrix into the interval $[1 / 9,9]$ and by Fedrizzi and Brunelli (2009) to define consistency-equivalence classes. 


\section{Axiom 4: Monotonicity on single comparisons}

Let us consider a consistent matrix with at least one non-diagonal entry $a_{p q} \neq 1$. If we increase or decrease the value of $a_{p q}$, and modify its reciprocal $a_{q p}$ accordingly, then the resulting matrix is not anymore consistent. In fact, in agreement with $\mathrm{A} 1$, the resulting matrix will have a degree of inconsistency which exceeds that of the consistent matrix. Axiom 4 (A4) establishes a condition of monotonicity for the inconsistency index with respect to single comparisons by requiring that the larger the change of $a_{p q}$ from its consistent value, the more inconsistent the resulting matrix will be. More formally, given a consistent matrix $\mathbf{A} \in \mathcal{A}^{*}$, let $\mathbf{A}_{p q}(\delta)$ be the inconsistent matrix obtained from $\mathbf{A}$ by replacing the entry $a_{p q}$ with $a_{p q}^{\delta}$, where $\delta \neq 1$. Necessarily, $a_{q p}$ must be replaced by $a_{q p}^{\delta}$ to preserve reciprocity. Let $\mathbf{A}_{p q}\left(\delta^{\prime}\right)$ be the inconsistent matrix obtained from $\mathbf{A}$ by replacing entries $a_{p q}$ and $a_{q p}$ with $a_{p q}^{\delta^{\prime}}$ and $a_{q p}^{\delta^{\prime}}$ respectively. A4 can then be formulated as

$$
\begin{aligned}
& \delta^{\prime}>\delta>1 \Rightarrow I\left(\mathbf{A}_{p q}\left(\delta^{\prime}\right)\right) \geq I\left(\mathbf{A}_{p q}(\delta)\right) \\
& \delta^{\prime}<\delta<1 \Rightarrow I\left(\mathbf{A}_{p q}\left(\delta^{\prime}\right)\right) \geq I\left(\mathbf{A}_{p q}(\delta)\right) .
\end{aligned}
$$

Axiom 4 can be equivalently formalized as follows.

Axiom 4. An inconsistency index I satisfies $A 4$, if and only if $I\left(\mathbf{A}_{p q}(\delta)\right)$ is a non-decreasing function of $\delta$ for $\delta>1$ and a non-increasing function of $\delta$ for $\delta<1$, for all the $\mathbf{A} \in \mathcal{A}^{*}$ and $p, q=1, \ldots, n$.

Example 4. Consider the consistent matrix

$$
\mathbf{A}=\left(\begin{array}{ccc}
1 & 2 & 4 \\
1 / 2 & 1 & 2 \\
1 / 4 & 1 / 2 & 1
\end{array}\right) \in \mathcal{A}^{*}
$$

Then, choosing, for instance, entry $a_{13}$ and changing its value and the value of its reciprocal accordingly, we obtain

$$
\mathbf{A}^{\prime}=\left(\begin{array}{ccc}
1 & 2 & 5 \\
1 / 2 & 1 & 2 \\
1 / 5 & 1 / 2 & 1
\end{array}\right) \quad \mathbf{A}^{\prime \prime}=\left(\begin{array}{ccc}
1 & 2 & 9 \\
1 / 2 & 1 & 2 \\
1 / 9 & 1 / 2 & 1
\end{array}\right)
$$

If an inconsistency index $I$ satisfies A4, then $I\left(\mathbf{A}^{\prime \prime}\right) \geq I\left(\mathbf{A}^{\prime}\right) \geq I(\mathbf{A})$, where the inequality between $I(\mathbf{A})$ and $I\left(\mathbf{A}^{\prime}\right)$ becomes strict if $\mathrm{A} 1$ holds. Note that, in this example, $\mathbf{A}^{\prime}=\mathbf{A}_{13}(\delta)$ with $\delta=\log _{4} 5$ and $\mathbf{A}^{\prime \prime}=\mathbf{A}_{13}\left(\delta^{\prime}\right)$ with $\delta^{\prime}=\log _{4} 9$.

Moreover, we note that A4 formalizes a property proved by Aupetit and Genest (1993) for Saaty's Consistency Index and considered by the authors as a necessary property. Furthermore, the case of a potentially consistent matrix with one deviating comparison was considered by Bryson (1995) in a property that he called 'single outlier neutralization' and by Choo and Wedley (2004) in their comparative study of methods to elicit the weight vector. A4 is also in the spirit of other known axiomatic systems. As examples, Cook and Kress (1988) considered two matrices differing by only one comparison and Kemeny and Snell (1962) proposed a similar axiomatic assumption for the distance between rankings. 


\section{Axiom 5: Continuity}

As defined in (5), an inconsistency index $I(\mathbf{A})$ is a function of $\mathbf{A} \in \mathcal{A}$. With this fifth axiom (A5), the continuity of the function is required in the set $\mathcal{A}$. More precisely, an index $I(\mathbf{A})$ is considered as a function of the $\left(\begin{array}{l}n \\ 2\end{array}\right)$ variables $a_{i j}, i<j$ and continuity of $I(\mathbf{A})$ is meant as the continuity of a function of $\left(\begin{array}{l}n \\ 2\end{array}\right)$ real variables. Axiom 5 can be formalized as follows

Axiom 5. An inconsistency index $I(\mathbf{A})$ satisfies $A 5$ if and only if it is a continuous function of the entries $a_{i j}$ of $\mathbf{A}$, with $a_{i j}>0, a_{i j} a_{j i}=1 \forall i, j$.

The importance of continuity in mathematical modelling has origins in the fact that it guarantees that infinitesimal variations in the input only generates an infinitesimal variation of the output, thus excluding functions with 'jumps'.

\subsection{Significance of the axioms}

Let us briefly discuss the necessity of the five axioms by showing that their violation could result in an unreasonable inconsistency measurement:

- If A1 is violated, two perfectly consistent matrices can have two different numerical consistency evaluations.

- If A2 is violated, different consistency evaluations could be associated to the same set of preferences, simply by renaming of alternatives.

- The effect of violation of A3 is apparent from Example 3. If A3 is not respected, matrix $\mathbf{A}(3)$, where inconsistent preferences are reinforced, could be evaluated less inconsistent than A.

- Let us consider Example 4 to show the necessity of A4. In the Example, matrix $\mathbf{A}^{\prime \prime}$ clearly differs from the consistent matrix $\mathbf{A}$ more than $\mathbf{A}^{\prime}$ does. As a consequence, $\mathbf{A}^{\prime \prime}$ cannot be evaluated less inconsistent than $\mathbf{A}^{\prime}$.

- As stated by Barzilai (1998), continuity 'is a reasonable requirement of any measure of amount of inconsistency'. In the proof of Proposition 5, referring to Barzilai's own index, we will show that a discontinuous index may assign the largest inconsistency evaluation to a matrix which is arbitrarily close to a consistent one.

In spite of the reasonability of A1-A5, they could be suspected of being too weak in order to characterize an inconsistency index. On the contrary, they turn out to be strictly demanding, since Propositions 5, 6, 7, and 8 will surprisingly show that they are not satisfied by four indices based on seemingly reasonable definitions.

\subsection{Logical consistency and independence}

A natural question is whether the axiomatic properties A1-A5 form an axiomatic system or not. In fact, in an axiomatic system, the axioms must be consistent (in a logical sense) and independent. The existence of, at least, one index satisfying A1-A5 proves that the axiomatic system is not logically contradictory and therefore the system is logically consistent. Another important result regards the independence of the axioms. Proving the independence would show that the axioms are not redundant, and therefore all of them shall be considered necessary.

Theorem 1. Axiomatic properties A1-A5 are logically consistent and independent. 
Proof. Propositions 1, 22 and 3 in Section 5 state that indices $C I, C I^{*}$ and $G C I$, respectively, satisfy all the five properties. Then, the axiomatic properties A1-A5 are logically consistent. Independence of a given axiom can be shown by providing an example of index satisfying all axioms except the one at stake. To prove the independence of A1, one can consider the following ad hoc constructed index,

$$
I_{1}(\mathbf{A})=\max \left\{C I^{*}(\mathbf{A})-1,0\right\} .
$$

As a consequence of Proposition 2, index $I_{1}$ satisfies A2-A5. Nevertheless, $I_{1}$ assigns value 0 also to some inconsistent pairwise comparison matrices, so that it does not satisfy A1. To prove independence of A2, one could instead consider

$$
I_{2}(\mathbf{A})=\sum_{i=1}^{n-2} \sum_{j=i+1}^{n-1} \sum_{k=j+1}^{n}\left(\frac{a_{i k}}{a_{i j} a_{j k}}+\frac{a_{i j} a_{j k}}{a_{i k}}-2\right) w_{i j k}
$$

with $w_{i j k}>0 \forall i, j, k, \quad 0<i<j<k \leq n$ and $w_{i j k} \neq w_{i^{\prime} j^{\prime} k^{\prime}}$ for some $0<i<j<k \leq n$ and $0<i^{\prime}<j^{\prime}<k^{\prime} \leq n$. Index $I_{2}$ does not satisfy A2, due to the presence of $w_{i j k}$. Conversely, it satisfies all the other axioms, the proof being similar to that of Proposition 2. Independence of A3 directly follows from Proposition 7. To prove independence of A4, we propose the following index,

$$
I_{4}(\mathbf{A})=\left(\max _{i \neq j} \max \left\{a_{i j}, a_{j i}\right\}-\min _{i \neq j} \max \left\{a_{i j}, a_{j i}\right\}+\epsilon\right) \times\left(C I^{*}(\mathbf{A})\right)^{0.1} .
$$

It can be proved that $I_{4}$ fails to satisfy A4 for a convenient choice of $\epsilon>0$ and A. Finally, it is easy to check that

$$
I_{5}(\mathbf{A})= \begin{cases}0 & \text { if } \mathbf{A} \in \mathcal{A}^{*}, \\ 1 & \text { if } \mathbf{A} \notin \mathcal{A}^{*}\end{cases}
$$

satisfies axioms A1-A4, but is not continuous and therefore does not fulfill A5, thus showing its independence.

Next, we shall investigate if existing inconsistency indices - especially those defined in the previous section - satisfy the axioms A1-A5.

\section{On the satisfaction of the axioms}

We first consider three inconsistency indices and prove that they satisfy all the axioms. Only later, we shall prove that some others do not satisfy some axioms. As anticipated in the introduction, most of the proofs are given in the appendix in order to simplify the description.

Saaty's $C I$ and indices $C I^{*}$ and $G C I$ satisfy the five axioms A1-A5. We can formalize it in the following propositions.

Proposition 1. Saaty's Consistency Index CI (6) satisfies the five axioms A1-A5.

Proposition 2. Index $C I^{*}$ satisfies the five axioms A1-A5.

Proposition 3. The Geometric Consistency Index GCI satisfies the five axioms A1-A5.

Let us now consider Barzilai's inconsistency index $R E$ and formulate the following interesting result,

Proposition 4. Let $\mathbf{A} \in \mathcal{A}$ and $\mathbf{A}(b)=\left(a_{i j}^{b}\right)_{n \times n}$. Then $R E(\mathbf{A})=R E(\mathbf{A}(b)) \forall b \neq 0$ and therefore $R E$ is invariant w.r.t. $f\left(a_{i j}\right)=a_{i j}^{b} \forall b>0$. 
Proposition 4 could be seen as a restriction of A3, where an inconsistency index is required to be invariant under function $f\left(a_{i j}\right)=a_{i j}^{b}$. Clearly, this implies that index $R E$ satisfies A3. The general result on index $R E$ is stated by the following proposition

Proposition 5. Index RE satisfies A1-A3, but it does not satisfy A4 and A5.

Note that Proposition 5 disproves the continuity of $R E$ claimed in the original paper by Barzilai (1998).

The following proposition concerns index $N I_{n}^{\sigma}$, introduced by Ramík and Korviny (2010), see definition 7. It remains unproved whether $N I_{n}^{\sigma}$ satisfies A3 or not.

Proposition 6. Index $N I_{n}^{\sigma}$ satisfies axioms A1, A2 and A5 but it does not satisfy A4.

Proof. The proof that $N I_{n}^{\sigma}$ satisfies axioms A1 and A2 is straightforward. To prove that $N I_{n}^{\sigma}$ does not satisfy A4, let us consider the following consistent pairwise comparison matrix,

$$
\mathbf{A}=\left(\begin{array}{cccc}
1 & 1 / 3 & 1 / 3 & 1 / 9 \\
3 & 1 & 1 & 1 / 3 \\
3 & 1 & 1 & 1 / 3 \\
9 & 3 & 3 & 1
\end{array}\right) \in \mathcal{A}^{*}
$$

If entry $a_{14}$ is changed, and its reciprocal $a_{41}$ varies accordingly, then, the violation of A4 can be appreciated in Figure 1, where $N I_{n}^{\sigma}(\mathbf{A})$ is plotted as a function of $a_{14}$, being $n=4$ and $\sigma=9$. In fact, A4 implies that such a function should be monotonically increasing for $a_{14}>1 / 9$, but

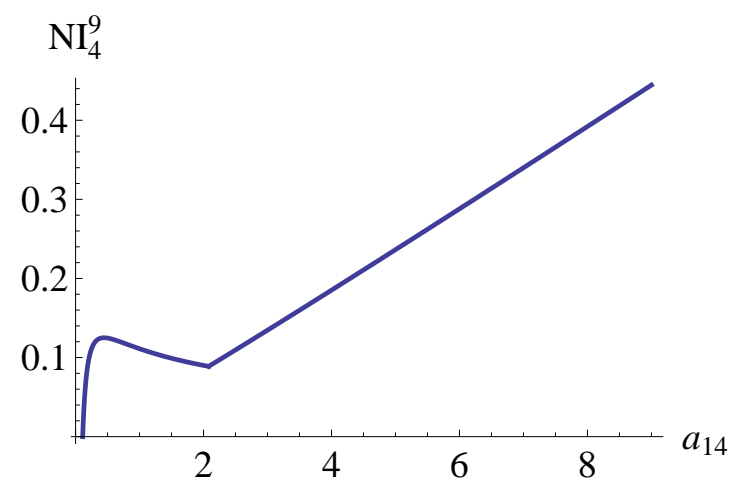

Figure 1: $N I_{4}^{9}(\mathbf{A})$ as a function of $a_{14}$

this is not the case in this example, since, e.g., $2>0.5$ but the value of $N I_{4}^{9}(\mathbf{A})$ corresponding to $a_{14}=2$ is smaller than the value of $N I_{4}^{9}(\mathbf{A})$ corresponding to $a_{14}=0.5$. Continuity of $N I_{n}^{\sigma}$ follows from continuity of $\max \{\cdot, \cdot\}$, so that A5 is satisfied.

We can only conjecture that the behavior described in Figure 1 is related with the fact, noted by Brunelli (2011), that index $N I_{n}^{\sigma}$ fails to identify the most inconsistent $3 \times 3$ matrix.

We next consider another index which fails to satisfy one of the axioms: the Harmonic Consistency Index (12).

Proposition 7. Index HCI satisfies A1, A2, A4 and A5 but it does not satisfy A3.

The following example derives from the proof of proposition 7 and is aimed to clarify the behavior of $H C I$ and to show the importance of A3. 
Example 5. Consider the following matrix $\mathbf{A}$ and its derived matrix $\mathbf{A}(b)=\left(a_{i j}^{b}\right)$

$$
\mathbf{A}=\left(\begin{array}{cccc}
1 & 4 & 1 / 2 & 2 \\
1 / 4 & 1 & 1 / 4 & 2 \\
2 & 4 & 1 & 2 \\
1 / 2 & 1 / 2 & 1 / 2 & 1
\end{array}\right) \quad \mathbf{A}(b)=\left(\begin{array}{cccc}
1^{b} & 4^{b} & (1 / 2)^{b} & 2^{b} \\
(1 / 4)^{b} & 1^{b} & (1 / 4)^{b} & 2^{b} \\
2^{b} & 4^{b} & 1^{b} & 2^{b} \\
(1 / 2)^{b} & (1 / 2)^{b} & (1 / 2)^{b} & 1
\end{array}\right)
$$

It is possible to illustrate the behavior of $H C I(\mathbf{A}(b))$ by means of Figure 2, The index initially increases, but then it decreases and converges to full $H C I$-consistency as $b$ grows.

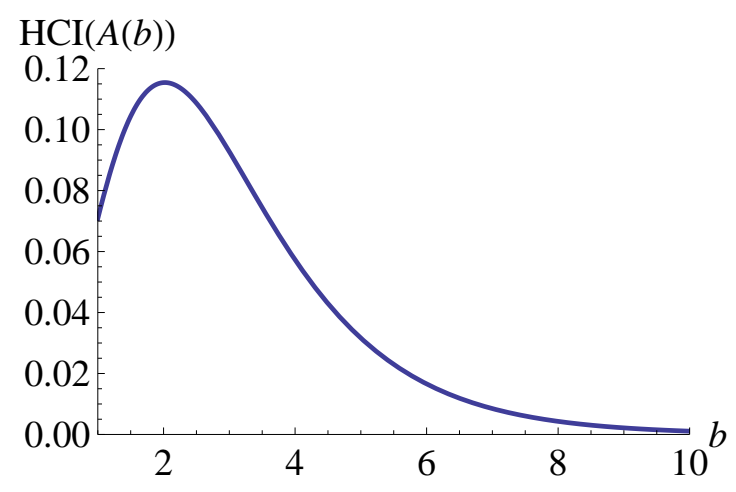

Figure 2: Index $H C I(\mathbf{A}(b))$ as a function of $b$

The last index considered in this section is the index $G W$ of Golden and Wang (1989) and the following proposition states the corresponding results. It remains unproved whether $G W$ satisfies A4 or not.

Proposition 8. Index $G W$ satisfies A1, A2 and A5. If the priority vector is computed by means of the geometric mean method, then index $G W$ does not satisfy A3.

The following example derives from the proof of Proposition 8 and, similarly to example 5 , shows the convergence to zero of the index $G W$.

Example 6. Consider the pairwise comparison matrix $\mathbf{A}(b)$ obtained from $\mathbf{A}$ as

$$
\mathbf{A}=\left(\begin{array}{cccc}
1 & 3 & 1 / 4 & 2 \\
1 / 3 & 1 & 1 / 7 & 2 \\
4 & 7 & 1 & 6 \\
1 / 2 & 1 / 2 & 1 / 6 & 1
\end{array}\right) \quad \mathbf{A}(b)=\left(\begin{array}{cccc}
1^{b} & 3^{b} & (1 / 4)^{b} & 2^{b} \\
(1 / 3)^{b} & 1^{b} & (1 / 7)^{b} & 2^{b} \\
4^{b} & 7^{b} & 1^{b} & 6^{b} \\
(1 / 2)^{b} & (1 / 2)^{b} & (1 / 6)^{b} & 1^{b}
\end{array}\right)
$$

and note that the third row of $\mathbf{A}$ contains all the greatest elements of each column. It is possible to plot the behavior of $G W$ and obtain the graph in Figure 3, which represents the $G W$-inconsistency of matrix $\mathbf{A}(b)$ in (23).

Finally, Table 1 summarizes the findings obtained in this section.

\section{Discussion and Future Research}

Propositions 6, 7 and 8, together with the corresponding examples, suggest that A3 and A4 are the most demanding axioms. Let us make some other remarks to clarify A3 and A4. First, we 


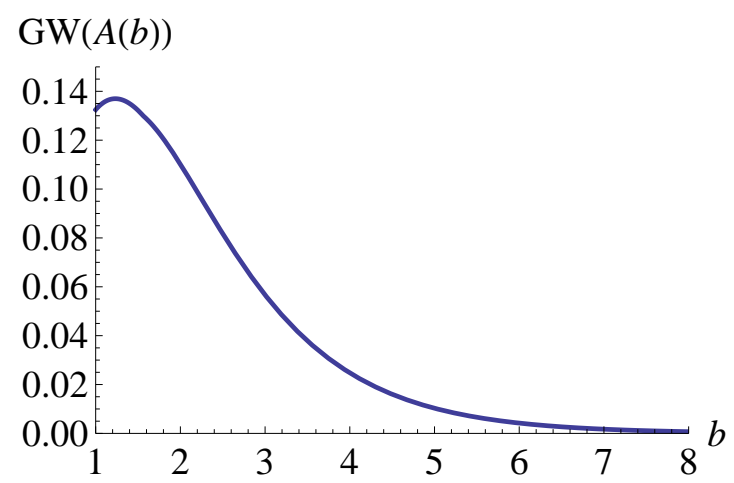

Figure 3: Index $G W(\mathbf{A}(b))$ as a function of $b$

\begin{tabular}{l|ccccc} 
& $\mathrm{A} 1$ & $\mathrm{~A} 2$ & $\mathrm{~A} 3$ & $\mathrm{~A} 4$ & $\mathrm{~A} 5$ \\
\hline$C I$ (def. 11) & $\mathrm{Y}$ & $\mathrm{Y}$ & $\mathrm{Y}$ & $\mathrm{Y}$ & $\mathrm{Y}$ \\
$G W$ (def. 21) & $\mathrm{Y}$ & $\mathrm{Y}$ & $\mathrm{N}$ & $?$ & $\mathrm{Y}$ \\
$G C I$ (def. 33) & $\mathrm{Y}$ & $\mathrm{Y}$ & $\mathrm{Y}$ & $\mathrm{Y}$ & $\mathrm{Y}$ \\
$R E$ (def. [4) & $\mathrm{Y}$ & $\mathrm{Y}$ & $\mathrm{Y}$ & $\mathrm{N}$ & $\mathrm{N}$ \\
$C I^{*}$ (def. 55) & $\mathrm{Y}$ & $\mathrm{Y}$ & $\mathrm{Y}$ & $\mathrm{Y}$ & $\mathrm{Y}$ \\
$H C I$ (def. 61) & $\mathrm{Y}$ & $\mathrm{Y}$ & $\mathrm{N}$ & $\mathrm{Y}$ & $\mathrm{Y}$ \\
$N I_{n}^{\sigma}$ (def. 7) & $\mathrm{Y}$ & $\mathrm{Y}$ & $?$ & $\mathrm{~N}$ & $\mathrm{Y}$
\end{tabular}

Table 1: Summary of propositions: $\mathrm{Y}=$ axiom is satisfied, $\mathrm{N}=$ axiom is not satisfied, $?=$ unknown

propose a geometrical interpretation that could be useful to emphasize the role of A4 in requiring the non-decreasing property of an inconsistency index when moving away from consistency. Let us represent a consistent matrix $\mathbf{A} \in \mathcal{A}^{*}$ as a point in the Cartesian space $\mathbb{R}^{n(n-1) / 2}$, where the dimension $n(n-1) / 2$ is the number of upper-diagonal elements which are necessary and sufficient to identify a pairwise comparison matrix of order $n$. By increasing (decreasing) entry $a_{p q}$, the point $\mathbf{A}$ departs from set $\mathcal{A}^{*}$ and moves in the direction of the corresponding axis. Thus, A4 requires that an inconsistency index does not decrease whenever $\mathbf{A}$ moves away from the initial consistent position, in any of the $n(n-1) / 2$ possible directions. On the other hand, by referring to the same geometrical representation in the Cartesian space, the type of translation of point $\mathbf{A}$ induced by $\mathrm{A} 3$ is different from the one induced by A4, so that the joint effect is more general than the single ones.

In decision problems based on pairwise comparisons there are two phases: preference elicitation and priority vector computation. In the previous sections we defined and studied five axioms characterizing the inconsistency evaluation of the preferences elicited by a decision maker independently from the method used in deriving the priority vector. Therefore, we focused on pairwise comparison matrix $\mathbf{A}$ and the property of transitivity $a_{i k}=a_{i j} a_{j k} \forall i, j, k$. Nevertheless, by considering that consistency of a pairwise comparison matrix can equivalently be characterized by property $a_{i j}=w_{i} / w_{j} \forall i, j$, it is possible and relevant to study also the relationship between the inconsistency indices and the methods used for priority vector computation. Other investigations will add new insight on the relationship between the inconsistency indices and the methods used for computing priority vectors. 


\subsection{Conclusions}

The purpose of this paper was to introduce some formal order in the topic of consistency evaluation for pairwise comparison matrices. We proposed few and simply justifiable axiomatic properties to characterize inconsistency indices, discovering that some indices proposed in the literature fail to satisfy some properties. We hope that our proposal will open a debate and stimulate further studies.

\section{References}

Aczel J (1966). Lectures on Functional Equations and their Applications. Academic Press: New York.

Aguaròn, J and Moreno-Jimènez, J M (2003). The geometric consistency index: Approximated threshold. European Journal of Operational Research 147(1): 137-145.

Aupetit B and Genest C (1993). On some useful properties of the Perron eigenvalue of a positive reciprocal matrix in the context of the analytic hierarchy process. European Journal of Operational Research 70(2): 263-268

Barzilai J (1998). Consistency measures for pairwise comparison matrices. Journal of Multi-Criteria Decision Analysis 7(3): 123-132.

Bozóki S and Rapcsák T (2008). On Saaty's and Koczkodaj's inconsistencies of pairwise comparison matrices. Journal of Global Optimization 42(2): 157-175.

Bozóki S, Fülöp J and Rónyai L (2010). On optimal completion of incomplete pairwise comparison matrices. Mathematical and Computer Modelling 52(1-2): 318-333.

Brunelli M (2011). A note on the article "Inconsistency of pair-wise comparison matrix with fuzzy elements based on geometric mean" [Fuzzy Sets and Systems 161 (2010) 1604-1613]. Fuzzy Sets and Systems 176(1): 76-78.

Brunelli M, Canal L and Fedrizzi M (2013a). Inconsistency indices for pairwise comparison matrices: a numerical study. Annals of Operations Research doi: 10.1007/s10479-013-1329-0.

Brunelli M, Critch A and Fedrizzi M (2013b). A note on the proportionality between some consistency indices in the AHP. Applied Mathematics and Computation 219(14): 7901-7906.

Bryson N (1995). A goal programming method for generating priority vectors. Journal of the Operational Research Society 46(5): 641-648.

Cavallo B and D'Apuzzo L (2009). A general unified framework for pairwise comparison matrices in multicriterial methods. International Journal of Intelligent Systems 24(4): 377-398.

Choo E U and Wedley W C (2004). A common framework for deriving preference values from pairwise comparison matrices. Computers \& Operations Research 31(6): 893-908.

Cook W D and Kress M (1988). Deriving weights from pairwise comparison ratio matrices: An axiomatic approach. European Journal of Operational Research 37(3): 355-362.

Crawford G and Williams C (1985). A note on the analysis of subjective judgement matrices. Journal of Mathematical Psychology 29(4): 25-40.

Duszak Z and Koczkodaj W W (1994). Generalization of a new definition of consistency for pairwise comparisons. Information Processing Letters 52(5): 273-276.

Fedrizzi M and Brunelli M (2009). Fair consistency evaluation in reciprocal relations and group decision making. New Mathematics and Natural Computation 5(2): 407-420.

Golden B L and Wang Q (1989). An alternate measure of consistency. In: Golden B L., Wasil E A and Harker P T (eds), The Analytic Hierarchy Process, Applications and studies, (pp. 68-81), Springer-Verlag: BerlinHeidelberg. 
Harker P T (1987). Incomplete pairwise comparisons in the analytic hierarchy process. Mathematical Modelling 9(11): $837-848$.

Harker P T, Vargas L G (1987). The theory of ratio scale estimation: Saaty's Analytic Hierarchy Process. Management Science 33(11): 1383-1403.

Herrera-Viedma E, Herrera F, Chiclana F and Luque M (2004). Some issues on consistency of fuzzy preference relations. European Journal of Operational Research 154(1): 98-109.

Horn R A and Johnson C R (1985). Matrix Analysis, Cambridge University Press: New York.

Irwin F W (1958). An analysis of the concepts of discrimination and preference. The American Journal of Psychology 71(1): 152-163.

Ishizaka A (2011). Does AHP help us make a choice? An experimental evaluation. Journal of the Operational Research Society 62(10): 1801-1812.

Ishizaka A and Labib A (2011). Review of the main developments in the analytic hierarchy process. Expert Systems with Applications 38(11): 14336-14345.

Kemeny, J G and Snell J L (1962). Mathematical Models in the Social Sciences. Blaisdell: New York.

Kingman, J F C (1961). A convexity property of positive matrices. The Quarterly Journal of Mathematics. Oxford. Second Series 12(1), 283-284.

Koczkodaj W W (1993). A new definition of consistency of pairwise comparisons. Mathematical and Computer Modelling 18(7): 79-84.

Lamata M T and Peláez J I (2002). A method for improving the consistency of judgments. International Journal of Uncertainty, Fuzziness and Knowledge-Based Systems 10(6): 677-686.

Osei-Bryson N (2006). An action learning approach for assessing the consistency of pairwise comparison data. European Journal of Operational Research 174(1): 234-244.

Peláez J I and Lamata M T (2003). A new measure of inconsistency for positive reciprocal matrices. Computer and Mathematics with Applications 46(12): 1839-1845.

Ramík J and Korviny P (2010). Inconsistency of pair-wise comparison matrix with fuzzy elements based on geometric mean. Fuzzy Sets and Systems 161(11): 1604-1613.

Saaty T L (1977). A scaling method for priorities in hierarchical structures. Journal of Mathematical Psychology 15(3): $234-281$.

Saaty T L (1993). What is relative measurement? The ratio scale phantom. Mathematical and Computer Modelling 17(4-5): $1-12$.

Saaty T L (1994). Highlights and critical points in the theory and application of the Analytic Hierarchy Process. European Journal of Operational Research 74(3): 426-447.

Salo A A (1993). Inconsistency analysis by approximately specified priorities. Mathematical and Computer Modelling 17(4-5): 123-133.

Shiraishi S, Obata T and Daigo M (1998). Properties of a positive reciprocal matrix and their application to AHP. Journal of the Operations Research Society of Japan 41(3): 404-414.

Shiraishi S, Obata T, Daigo M and Nakajima N (1999). Assessment for an incomplete matrix and improvement of the inconsistent comparison: computational experiments. Proceedings of ISAHP 1999, Kobe, Japan

Stein W E and Mizzi P J (2007). The harmonic consistency index for the analytic hierarchy process. European Journal of Operational Research 177(1): 488-497.

Xu Z and Cuiping W (1999). A consistency improving method in the analytic hierarchy process. European Journal of Operational Research 116(2): 443-449.

Xu Z and Xia M (1999). Iterative algorithms for improving consistency of intuitionistic preference relations. Journal of Operational Research Society doi: 10.1057/jors.2012.178. 


\section{Appendix}

Proof of Proposition 1. In the following we prove each axiom separately.

A1 This was proved already by Saaty (1977).

A2 It is known that the characteristic polynomial of a matrix $\mathbf{A}$ equals the characteristic polynomial of the matrix $\mathbf{S}^{-1} \mathbf{A S}$ where $\mathbf{S}$ is any non-singular matrix and $\mathbf{S}^{-1}$ its inverse (see Horn and Johnson (1985), p. 45). We also know that any permutation matrix is an orthogonal matrix, and therefore its inverse is its transpose. Thus, using the notation used in (15), we also know that the characteristic polynomial of $\mathbf{A}$ is the same of $\mathbf{P A P} \mathbf{P}^{\mathrm{T}}$. This implies that the index $C I$ remains unchanged.

A3 The proof relies on a theorem from linear algebra by Kingman (1961) stating that if the elements $a_{i j}(b)$ of a matrix $\mathbf{A}$ are logconvex functions of $b$, then the maximum eigenvalue $\lambda_{\max }(\mathbf{A})$ is a logconvex (and hence convex) function of $b$. Given a pairwise comparison matrix $\mathbf{A}=\left(a_{i j}\right) \in \mathcal{A}$, let us apply the preference intensifying function $f\left(a_{i j}\right)=a_{i j}^{b}$. The entries of the obtained matrix $\mathbf{A}(b)=\left(a_{i j}(b)\right)_{n \times n}=\left(a_{i j}^{b}\right)_{n \times n}$ are logconvex functions of parameter $b$ (see Bozóki et al. 2010). From the theorem, $\lambda_{\max }(\mathbf{A}(b))$ is a convex function of $b$. Moreover, $\lambda_{\max }(\mathbf{A}(b))$ reaches its minimum value $n$ for $b=0$, since $\mathbf{A}(0)$ is the consistent matrix with all entries equal to one (indifference matrix). It follows that $\lambda_{\max }(\mathbf{A}(b))$ is a non-decreasing function for $b \geq 0$ and hence, in particular, for $b \geq 1$. The same applies to $C I$, being an increasing affine transform of $\lambda_{\max }(\mathbf{A}(b))$. Therefore, $C I$ satisfies (19)).

A4 It was proved by Aupetit and Genest (1993), that $\lambda_{\max }$ must be either increasing, decreasing or U-shaped as a function of a single upper triangular entry, say $a_{p q}$, of a positive reciprocal matrix. Let $\mathbf{A}$ be a consistent matrix, $\mathbf{A} \in \mathcal{A}^{*}$ and let $a_{p q} \neq 1$ be an arbitrarily fixed entry of $\mathbf{A}$. Then it is $\lambda_{\max }(\mathbf{A})=n$. Using the notation of section 4, let $\mathbf{A}_{p q}(\delta)$ be the inconsistent matrix obtained from $\mathbf{A}$ by replacing $a_{p q}$ with with a different value $a_{p q}^{\delta}>0$, being $a_{q p}$ consequently replaced with $1 / a_{p q}^{\delta}$. Then $\lambda_{\max }$ becomes larger than $n$, $\lambda_{\max }\left(\mathbf{A}_{p q}(\delta)\right) \geq n$. That is, $\lambda_{\max }\left(\mathbf{A}_{p q}(\delta)\right)$ reaches its minimum value $n$ when $a_{p q}^{\delta}=a_{p q}$. It follows that $\lambda_{\max }\left(\mathbf{A}_{p q}(\delta)\right)$ is U-shaped as a function of $a_{p q}^{\delta}$, reaching its minimum value for $a_{p q}^{\delta}=a_{p q}$. The same applies to $C I$, being an increasing affine transform of $\lambda_{\max }$, which proves that A4 is satisfied.

A5 Continuity holds due to the continuous dependence of the zeroes of a polynomial on its coefficients

Proof of Proposition 2. We shall proceed by proving that it satisfies each axiom:

A1 This axiom is satisfied by $C I^{*}$ as is can be proved that the non-negative quantity

$$
\frac{a_{i k}}{a_{i j} a_{j k}}+\frac{a_{i j} a_{j k}}{a_{i k}}-2
$$

reaches its minimum, which is zero, if and only if $a_{i k}=a_{i j} a_{j k}$. The proof is based on the study of the function $G(x)=x+\frac{1}{x}-2$ where $x=\frac{a_{i k}}{a_{i j} a_{j k}}$. Considering $x>0$, then $G(x)$ reaches its minimum for $x=1$. This implies $a_{i k}=a_{i j} a_{j k}$.

A2 Observe that: (i) expression (11) considers all the triplets $\left\{x_{i}, x_{j}, x_{k}\right\}$ only once; (ii) given a triplet $\left\{x_{i}, x_{j}, x_{k}\right\}$, the terms in (11) corresponding to these indices are independent from 
the order of the indices, that is

$$
\frac{a_{i k}}{a_{i j} a_{j k}}+\frac{a_{i j} a_{j k}}{a_{i k}}=\frac{a_{\pi(i) \pi(k)}}{a_{\pi(i) \pi(j)} a_{\pi(j) \pi(k)}}+\frac{a_{\pi(i) \pi(j)} a_{\pi(j) \pi(k)}}{a_{\pi(i) \pi(k)}}
$$

holds for any permutation map $\pi$.

A3 It is possible to prove that each term of the sum (11) corresponding to an inconsistent triplet increases if we apply $f\left(a_{i j}\right)=a_{i j}^{b}, b>1$. In fact, applying $f$ one obtains:

$$
\frac{a_{i k}^{b}}{a_{i j}^{b} a_{j k}^{b}}+\frac{a_{i j}^{b} a_{j k}^{b}}{a_{i k}^{b}}-2=\left(\frac{a_{i k}}{a_{i j} a_{j k}}\right)^{b}+\left(\frac{a_{i j} a_{j k}}{a_{i k}}\right)^{b}-2 .
$$

It is then necessary to prove that (25) is increasing with respect to $b>1$. Let us recall that $G(x)=x+\frac{1}{x}-2$ and $x=\frac{a_{i k}}{a_{i j} a_{j k}}$. Let

$$
g(x, b)=x^{b}+\frac{1}{x^{b}}-2 .
$$

We then need to prove that $g(x, b)$ is increasing with respect to $b>1$, that is

$$
b_{2}>b_{1} \Rightarrow g\left(x, b_{2}\right) \geq g\left(x, b_{1}\right)
$$

for all $x>0$. It is

$$
\frac{\partial g(x, b)}{\partial b}=\frac{\partial x^{b}}{\partial b}+\frac{\partial x^{-b}}{\partial b}=x^{b} \ln x-x^{-b} \ln x=(\ln x)\left(x^{b}-\frac{1}{x^{b}}\right) .
$$

To study the sign of $\frac{\partial g(x, b)}{\partial b}$, one can more simply analyze $h(z)=z-\frac{1}{z}$ in $] 0, \infty[$, where $z=x^{b}$

$$
h(z)=z-\frac{1}{z}=\frac{z^{2}-1}{z} .
$$

Then $0<z<1 \Rightarrow h(z)<0$, and $z>1 \Rightarrow h(z)>0$. Consequently, for $b>1$,

$$
\begin{aligned}
0<x<1 & \Rightarrow \ln x<0 \\
& \Rightarrow 0<z=x^{b}<1 \Rightarrow h(z)=\left(x^{b}-\frac{1}{x^{b}}\right)<0 \\
& \Rightarrow \frac{\partial g(x, b)}{\partial b}=(\ln x)\left(x^{b}-\frac{1}{x^{b}}\right)>0 \\
x>1 & \Rightarrow \ln x>0 \\
& \Rightarrow z=x^{b}>1 \Rightarrow h(z)=\left(x^{b}-\frac{1}{x^{b}}\right)>0 \\
& \Rightarrow \frac{\partial g(x, b)}{\partial b}=(\ln x)\left(x^{b}-\frac{1}{x^{b}}\right)>0 .
\end{aligned}
$$

Finally, if $x=1$, then

$$
\frac{\partial g(1, b)}{\partial b}=0
$$


Therefore, $g(x, b)$ is increasing respect to $b>0$ for $x>0 x \neq 1$. For $x=1$, representing consistent triples, we get $g(1, b)=0 \forall b$.

To conclude, each term of the sum (11) corresponding to an inconsistent triplet increases by applying function $f$ with $b>1$, hence

$$
\begin{aligned}
& C I^{*}(\mathbf{A})=0 \quad \Rightarrow \quad C I^{*}(\mathbf{A}(b))=C I^{*}(\mathbf{A})=0 \\
& C I^{*}(\mathbf{A})>0 \Rightarrow C I^{*}(\mathbf{A}(b))>C I^{*}(\mathbf{A}) .
\end{aligned}
$$

A4 Without loss of generality, let us fix the entry $a_{p q}$ of $\mathbf{A}$ with $p<q$, and replace $a_{p q}$ with $a_{p q}^{\delta}$ and $a_{q p}$ with $a_{q p}^{b}$. Considering index (11) for the obtained matrix $\mathbf{A}_{p q}(\delta)$, some terms of the sum contain $a_{p q}^{\delta}$, while others do not. Those not containing $a_{p q}^{\delta}$ remain unchanged. Let us first consider the following terms of the sum:

$$
\frac{a_{p q}^{\delta}}{a_{p j} a_{j q}}+\frac{a_{p j} a_{j q}}{a_{p q}^{\delta}}-2 .
$$

Since $\mathbf{A}$ is consistent, we can set $x=a_{i k}=a_{i j} a_{j k}$ and rewrite (31) as a function of $x$ and $\delta$ :

$$
H(x, \delta)=\frac{x^{\delta}}{x}+\frac{x}{x^{\delta}}-2=x^{\delta-1}+x^{1-\delta}-2 .
$$

It is:

$$
\frac{\partial H(x, \delta)}{\partial \delta}=x^{\delta-1} \ln x-x^{1-\delta} \ln x=\underbrace{x^{1-\delta}}_{>0} \underbrace{\left(x^{2 \delta-2}-1\right)}_{\mathrm{I}} \underbrace{\ln x}_{\mathrm{II}} .
$$

Thus, as pairwise comparison matrices are positive, i.e. $x>0$, one obtains the following four cases

$$
\begin{aligned}
& \left.\begin{array}{l}
\delta>1 \\
x>1
\end{array}\right\} \Rightarrow\left\{\begin{array}{l}
\mathrm{I}>0 \\
\mathrm{II}>0
\end{array} \quad \Rightarrow \frac{\partial H}{\partial \delta}>0\right. \\
& \left.\begin{array}{r}
\delta>1 \\
0<x<1
\end{array}\right\} \Rightarrow\left\{\begin{array}{l}
\mathrm{I}<0 \\
\mathrm{II}<0
\end{array} \quad \Rightarrow \frac{\partial H}{\partial \delta}>0\right. \\
& \left.\begin{array}{l}
\delta<1 \\
x>1
\end{array}\right\} \Rightarrow\left\{\begin{array}{l}
\mathrm{I}<0 \\
\mathrm{II}>0
\end{array} \Rightarrow \frac{\partial H}{\partial \delta}<0\right. \\
& \left.\begin{array}{r}
\delta<1 \\
0<x<1
\end{array}\right\} \Rightarrow\left\{\begin{array}{l}
\mathrm{I}>0 \\
\mathrm{II}<0
\end{array} \Rightarrow \frac{\partial H}{\partial \delta}<0 .\right.
\end{aligned}
$$

Hence, every term of the sum (11) with the form (31) is an increasing function of $\delta$ for $\delta>1$ and decreasing function for $\delta<1$, thus satisfying (20). The other type of terms in the sum (11) containing $a_{p q}^{\delta}$ are those in the form

$$
\frac{a_{p k}}{a_{p q}^{\delta} a_{q k}}+\frac{a_{p q}^{\delta} a_{q k}}{a_{p k}}-2 .
$$

By means of a reasoning similar to the previous one, it can be proved that also (32) is an increasing function of $\delta$ for $\delta>1$ and decreasing function for $\delta<1$. To summarize, A4 is satisfied by index $C I^{*}$.

A5 For positive matrices, function $C I^{*}$ is continuous, as it is a sum of continuous functions. 
Proof of Proposition 3. We shall prove each axiom separately.

A1 It is satisfied, since it is $a_{i j}=w_{i} / w_{j} \forall i, j$ if and only if $\mathbf{A} \in \mathcal{A}^{*}$, with $G C I(\mathbf{A})=\nu=0$.

$\mathbf{A} 2$ The second axiom is verifiable by rewriting the index $G C I$ as

$$
G C I(\mathbf{A})=\frac{1}{(n-1)(n-2)} \sum_{i=1}^{n} \sum_{j=1}^{n} \ln ^{2}\left(a_{i j} \frac{w_{j}}{w_{i}}\right) .
$$

A3 We consider $\mathbf{A}(b)=\left(a_{i j}^{b}\right)_{n \times n}$ and $w_{i}(\mathbf{A}(b))$ be the $i$-th weight obtained from $\mathbf{A}(b)$ by means of (3). Then, we obtain

$$
w_{i}(\mathbf{A}(b))=\left(\prod_{i=1}^{n} a_{i j}^{b}\right)^{\frac{1}{n}}=\left(\prod_{i=1}^{n} a_{i j}\right)^{\frac{b}{n}}=\left(w_{i}(\mathbf{A})\right)^{b},
$$

so that, considering the terms of the sum (9) for the matrix $\mathbf{A}(b)$, we can derive the following

$$
\ln ^{2}\left(a_{i j}^{b} \frac{w_{j}(\mathbf{A}(b))}{w_{i}(\mathbf{A}(b))}\right)=\ln ^{2}\left(a_{i j} \frac{w_{j}(\mathbf{A})}{w_{i}(\mathbf{A})}\right)^{b}=b^{2} \ln ^{2}\left(a_{i j} \frac{w_{j}}{w_{i}}\right)
$$

Hence,

$$
G C I(\mathbf{A}(b))=b^{2} G C I(\mathbf{A})
$$

and consequently A3 is satisfied, since

$$
G C I(\mathbf{A}(b)) \geq G C I(\mathbf{A}) \quad \forall b>1 .
$$

A4 As proved by Brunelli et al. (2013b), the index $G C I$ is proportional to the following quantity

$$
\sum_{i=1}^{n-2} \sum_{j=i+1}^{n-1} \sum_{k=j+1}^{n}\left(\log _{9} a_{i k} a_{k j} a_{j i}\right)^{2}
$$

Given a consistent matrix $\mathbf{A} \in \mathcal{A}^{*}$, let us fix the entry $a_{p q}(\neq 1)$ of $\mathbf{A}$ with $p<q$. We replace $a_{p q}$ with $a_{p q}^{\delta}$ and its reciprocal $a_{q p}$ with $a_{q p}^{\delta}$ obtaining the matrix $\mathbf{A}_{p q}(\delta)$. Quantity (33) is clearly null for $\mathbf{A}$, while for $\mathbf{A}_{p q}(\delta)$ only the terms not containing $a_{p q}^{\delta}$ or $a_{q p}^{\delta}$ are null. Hence, let us consider the terms of (33) for $\mathbf{A}_{p q}(\delta)$ containing $a_{p q}^{\delta}$ (results can be automatically extended to the terms containing $a_{q p}^{\delta}$ ):

$$
\left(\log _{9} a_{p q}^{\delta} a_{q j} a_{j p}\right)^{2} .
$$

Since $\mathbf{A}$ is consistent, it is $a_{p q}=a_{p j} a_{j q}$. Like in previous proofs, let us denote this quantity by $x$. Then, thanks to reciprocity, it is $a_{q j} a_{j p}=1 / x$ and thus

$$
\left(\log _{9} a_{p q}^{\delta} a_{q j} a_{j p}\right)^{2}=\left(\log _{9} a_{p q}^{\delta-1} a_{p q} a_{q j} a_{j p}\right)^{2}=\left(\log _{9} x^{\delta-1}\right)^{2} .
$$

Then every term in (33) containing $a_{p q}^{\delta}$ has the same expression, say $\beta(x, \delta)=\left(\log _{9} x^{\delta-1}\right)^{2}=$ $\left((\delta-1) \log _{9} x\right)^{2}$. Taking the derivative with respect to $\delta$, it is,

$$
\frac{\partial \beta(x, \delta)}{\partial \delta}=2(\delta-1)\left(\log _{9} x\right)^{2}
$$

and then

$$
\begin{aligned}
& \delta>1 \Rightarrow \frac{\partial \beta(x, \delta)}{\partial \delta}>0 \\
& \delta<1 \Rightarrow \frac{\partial \beta(x, \delta)}{\partial \delta}<0
\end{aligned}
$$

and the same holds also for the sum in (33), thus proving that $G C I$ satisfies A4. 
A5 For positive matrices, function $G C I$ is continuous, as it is a sum of continuous functions.

Proof of Proposition 不. For any $b \neq 0$ it is

$$
\begin{aligned}
R E(\mathbf{A}(b)) & =1-\frac{\sum_{i=1}^{n} \sum_{j=1}^{n}\left(\frac{1}{n} \sum_{k=1}^{n} \log a_{i k}^{b}-\frac{1}{n} \sum_{k=1}^{n} \log a_{j k}^{b}\right)^{2}}{\sum_{i=1}^{n} \sum_{j=1}^{n}\left(\log a_{i j}^{b}\right)^{2}} \\
& =1-\frac{\sum_{i=1}^{n} \sum_{j=1}^{n} b^{2}\left(\frac{1}{n} \sum_{k=1}^{n} \log a_{i k}-\frac{1}{n} \sum_{k=1}^{n} \log a_{j k}\right)^{2}}{\sum_{i=1}^{n} \sum_{j=1}^{n} b^{2}\left(\log a_{i j}\right)^{2}} \\
& =1-\frac{\sum_{i=1}^{n} \sum_{j=1}^{n}\left(\frac{1}{n} \sum_{k=1}^{n} \log a_{i k}-\frac{1}{n} \sum_{k=1}^{n} \log a_{j k}\right)^{2}}{\sum_{i=1}^{n} \sum_{j=1}^{n}\left(\log a_{i j}\right)^{2}} \\
& =R E(\mathbf{A}) .
\end{aligned}
$$

Proof of Proposition 5. In the original paper by Barzilai (1998), it was remarked that A1 is satisfied by $R E$. The proof of the satisfaction of A2 is also elementary and Proposition 4 proves the satisfaction of A3. In the following we shall prove separately that A4 and A5 do not hold.

A4 Following Barzilai (1998), let us consider the equivalent additive formulation of the pairwise comparison matrices and rewrite $R E$ in the form

$$
R E(\mathbf{A})=\frac{\sum_{i=1}^{n} \sum_{j=1}^{n} e_{i j}}{\sum_{i=1}^{n} \sum_{j=1}^{n} a_{i j}},
$$

where $e_{i j}=a_{i j}-c_{i j}, \quad c_{i j}=w_{i}-w_{j}$ and $w_{i}=\frac{1}{n} \sum_{j=1}^{n} a_{i j}$. We start from a consistent (in the additive sense) matrix $\mathbf{A}$ and replace the entry $a_{p q}$ with $a_{p q}^{\prime}=a_{p q}+x, x \neq 0$. Necessarily, $a_{q p}$ must be replaced by $a_{q p}^{\prime}=a_{q p}-x$ to preserve the additive reciprocity, i.e. the antisymmetry of $\mathbf{A}$. Let $\mathbf{A}^{\prime}=\mathbf{A}^{\prime}(x)=\left(a_{i j}^{\prime}\right)$ be the obtained inconsistent matrix. In order to evaluate $R E\left(\mathbf{A}^{\prime}\right)$, let us calculate

$$
\begin{aligned}
& w_{i}^{\prime}=w_{i} \quad \text { if } \quad i \neq p, i \neq q \\
& w_{p}^{\prime}=\frac{1}{n} \sum_{j=1}^{n} a_{p j}^{\prime}=\frac{1}{n} \sum_{j=1}^{n} a_{p j}+\frac{x}{n}=w_{p}+\frac{x}{n} \\
& w_{q}^{\prime}=\frac{1}{n} \sum_{j=1}^{n} a_{q j}^{\prime}=\frac{1}{n} \sum_{j=1}^{n} a_{q j}-\frac{x}{n}=w_{q}-\frac{x}{n} \\
& c_{i j}^{\prime}=c_{i j} \quad \text { if } \quad i, j \neq p, q \\
& c_{p j}^{\prime}=w_{p}^{\prime}-w_{j}^{\prime}=w_{p}+\frac{x}{n}-w_{j}=c_{p j}+\frac{x}{n} \quad \text { if } \quad j \neq p, j \neq q \\
& c_{p q}^{\prime}=w_{p}^{\prime}-w_{q}^{\prime}=w_{p}+\frac{x}{n}-\left(w_{q}-\frac{x}{n}\right)=w_{p}-w_{q}+\frac{2 x}{n}=c_{p q}+\frac{2 x}{n} \\
& c_{i p}^{\prime}=w_{i}^{\prime}-w_{p}^{\prime}=w_{i}-w_{p}-\frac{x}{n}=c_{i p}-\frac{x}{n} \quad \text { if } \quad i \neq p, j \neq q \\
& c_{q j}^{\prime}=w_{q}^{\prime}-w_{j}^{\prime}=w_{q}-\frac{x}{n}-w_{j}=c_{q j}-\frac{x}{n} \quad \text { if } \quad j \neq p, j \neq q \\
& c_{q p}^{\prime}=w_{q}^{\prime}-w_{p}^{\prime}=w_{q}-\frac{x}{n}-\left(w_{p}+\frac{x}{n}\right)=w_{q}-w_{p}-\frac{2 x}{n}=c_{q p}-\frac{2 x}{n} \\
& c_{i q}^{\prime}=w_{i}^{\prime}-w_{q}^{\prime}=w_{i}-w_{q}+\frac{x}{n}=c_{i q}+\frac{x}{n} \quad \text { if } \quad i \neq p, i \neq q .
\end{aligned}
$$


Therefore, the values $e_{i j}^{\prime}$ are

$$
\begin{aligned}
& e_{i j}^{\prime}=a_{i j}^{\prime}-c_{i j}^{\prime}=0 \quad \text { if } \quad i, j \neq p, q \\
& e_{p j}^{\prime}=a_{p j}^{\prime}-c_{p j}^{\prime}=a_{p j}-\left(c_{p j}+\frac{x}{n}\right)=e_{p j}-\frac{x}{n}=-\frac{x}{n} \quad \text { if } \quad j \neq p, q \\
& e_{p q}^{\prime}=a_{p q}^{\prime}-c_{p q}^{\prime}=a_{p q}+x-\left(c_{p q}+\frac{2 x}{n}\right)=e_{p q}+x-\frac{2 x}{n}=x-\frac{2 x}{n}=\frac{n-2}{n} x \\
& e_{q j}^{\prime}=a_{q j}^{\prime}-c_{q j}^{\prime}=a_{q j}-\left(c_{q j}-\frac{x}{n}\right)=e_{q j}+\frac{x}{n}=\frac{x}{n} \quad \text { if } \quad j \neq p, q \\
& e_{q p}^{\prime}=a_{q p}^{\prime}-c_{q p}^{\prime}=a_{q p}-x-\left(c_{q p}-\frac{2 x}{n}\right)=e_{q p}-x+\frac{2 x}{n}=-\frac{n-2}{n} x \\
& e_{i p}^{\prime}=a_{i p}^{\prime}-c_{i p}^{\prime}=a_{i p}-\left(c_{i p}-\frac{x}{n}\right)=e_{i p}+\frac{x}{n}=\frac{x}{n} \quad \text { if } \quad i \neq p, q \\
& e_{i q}^{\prime}=a_{i q}^{\prime}-c_{i q}^{\prime}=a_{i q}-\left(c_{i q}+\frac{x}{n}\right)=e_{i q}-\frac{x}{n}=-\frac{x}{n} \quad \text { if } \quad i \neq p, q,
\end{aligned}
$$

and, clearly,

$$
e_{i i}^{\prime}=0 \quad i=1, \ldots, n .
$$

The relative error $R E\left(\mathbf{A}^{\prime}\right)$ is then

$$
\begin{aligned}
R E\left(\mathbf{A}^{\prime}\right) & =\frac{\sum_{i=1}^{n} \sum_{j=1}^{n}\left(e_{i j}^{\prime}\right)^{2}}{\sum_{i=1}^{n} \sum_{j=1}^{n}\left(a_{i j}^{\prime}\right)^{2}} \\
& =\frac{\sum_{j \neq q, p}\left(e_{p j}^{\prime}\right)^{2}+\left(e_{p q}^{\prime}\right)^{2}+\sum_{j \neq p, q}\left(e_{q j}^{\prime}\right)^{2}+\left(e_{q p}^{\prime}\right)^{2}+\sum_{i \neq p, q}\left(e_{i p}^{\prime}\right)^{2}+\sum_{i \neq p, q}\left(e_{i q}^{\prime}\right)^{2}}{\sum_{i j}\left(a_{i j}^{\prime}\right)^{2}} \\
& =\frac{\sum_{j \neq p, q}\left(-\frac{x}{n}\right)^{2}+\left(\frac{n-2}{n} x\right)^{2}+\sum_{j \neq p, q}\left(\frac{x}{n}\right)^{2}+\left(-\frac{n-2}{n} x\right)^{2}+\sum_{i \neq p, q}\left(\frac{x}{n}\right)^{2}+\sum_{i \neq p, q}\left(-\frac{x}{n}\right)^{2}}{\sum_{i j}\left(a_{i j}\right)^{2}-\left(a_{p q}\right)^{2}-\left(a_{q p}\right)^{2}+\left(a_{p q}+x\right)^{2}+\left(a_{q p}-x\right)^{2}} \\
& =\frac{4(n-2)\left(\frac{x}{n}\right)^{2}+2\left(\frac{n-2}{n} x\right)^{2}}{\sum_{i j}\left(a_{i j}\right)^{2}+2 a_{p q} x+x^{2}-2 a_{q p} x+x^{2}} \\
& =\frac{2\left(n^{2}-2 n\right)}{n^{2}} \frac{x^{2}}{2 x^{2}+4 a_{p q} x+\sum_{i j}\left(a_{i j}\right)^{2}} \\
& =H_{n} \frac{x^{2}}{x^{2}+2 \alpha x+K},
\end{aligned}
$$

where

$$
H_{n}=\frac{\left(n^{2}-2 n\right)}{n^{2}}=1-\frac{2}{n} ; \quad K=\frac{1}{2} \sum_{i j}\left(a_{i j}\right)^{2} ; \quad \alpha=a_{p q}
$$

By disregarding the positive constant $H_{n}$ (for $n \geq 3$ ) and taking the derivative of $R E\left(\mathbf{A}^{\prime}\right)$ with respect to $x$ one obtains

$$
\begin{aligned}
\frac{\partial R E\left(\mathbf{A}^{\prime}\right)}{\partial x} & =\frac{2 x\left(x^{2}+2 \alpha x+K\right)-x^{2}(2 x+2 \alpha)}{\left(x^{2}+2 \alpha x+K\right)^{2}} \\
& =2 \frac{(2 \alpha x+(K-\alpha)) x}{\left(x^{2}+2 \alpha x+K\right)^{2}} .
\end{aligned}
$$

Assuming, without loss of generality, $\alpha<0$, it follows $\frac{\alpha-K}{2 \alpha}>0$. Then, $R E\left(\mathbf{A}^{\prime}\right)$ decreases in $(-\infty, 0)$, increases in $\left(0, \frac{\alpha-K}{2 \alpha}\right)$ and decreases in $\left(\frac{\alpha-K}{2 \alpha},+\infty\right)$. This means that if $a_{p q}$ is increased by $x$, the inconsistency index $R E\left(\mathbf{A}^{\prime}\right)$ does not monotonically increases in $(0,+\infty)$, so that A4 is not satisfied. 
A5 From Proposition 4 , it follows that $R E(\mathbf{A})=R E(\mathbf{A}(b)) \forall b \neq 0$. Then, for every $\mathbf{A} \in \mathcal{A}$ it holds

$$
\lim _{b \rightarrow 0} R E(\mathbf{A}(b))=R E(\mathbf{A}) .
$$

Conversely, it is

$$
R E\left(\lim _{b \rightarrow 0} \mathbf{A}(b)\right)=R E\left((1)_{n \times n}\right)=0,
$$

and therefore

$$
\lim _{b \rightarrow 0} R E(\mathbf{A}(b)) \neq R E\left(\lim _{b \rightarrow 0} \mathbf{A}(b)\right)
$$

for every inconsistent matrix $\mathbf{A}$. Then, $R E(\mathbf{A})$ is not continuous in $(1)_{n \times n}$. Note that, since (35) and (36) hold for every matrix $\mathbf{A} \in \mathcal{A}$, then, in every neighborhood of the consistent matrix $(1)_{n \times n}$ there exist matrices $\mathbf{A}$ with any possible value of $R E(\mathbf{A})$, even the highest one $R E(\mathbf{A})=1$.

Proof of Proposition 7. We shall prove each axiom separately.

A1 This comes along with the result proved by Stein and Mizzi (2007) that $H C I(\mathbf{A})=0$ if and only if $\mathbf{A}$ is consistent.

A2 A permutation of the alternatives corresponds to a row-column permutation on the pairwise comparison matrix. When columns of $\mathbf{A}$ are swapped this induces a permutation of the indices of $s_{1}, \ldots, s_{n}$. When rows are swapped, this produces a change in the order of the arguments of sums $\sum_{i=1}^{n} a_{i j} \forall j$. As both the harmonic mean (13) and the sum are commutative functions, A2 is satisfied.

A3 Let us prove it for a subset of inconsistent pairwise comparison matrices. We consider all the inconsistent pairwise comparison matrices which have one column, say $j^{*}$, with all entries smaller than one except for the diagonal element $a_{j^{*} j^{*}}$, i.e. $\exists j^{*}, a_{i j^{*}}<1 \forall i \neq j^{*}$. In this case, if we apply transformation $f\left(a_{i j}\right)=a_{i j}^{b}$ and let $b$ increase to $+\infty$, we obtain that all the values $s_{j}$ tend to infinite except $s_{j^{*}}$ which tends to 1 . Consequently, all the terms of the sum $\sum_{j=1}^{n} \frac{1}{s_{j}}$ converge to 0 , except $1 / s_{j^{*}}$ which, instead, converges to 1 , showing that $H M(\mathbf{A})$ will tend to $n$ and $H C I(\mathbf{A})$ to 0 . As the initial set of matrices was inconsistent and therefore their value of $H C I$ must have been positive, A3 is not satisfied.

A4 Consider that a consistent pairwise comparison matrix of order $n$ can be written as



Let us apply the exponential function to the element $a_{12}(\neq 1)$ and its reciprocal:

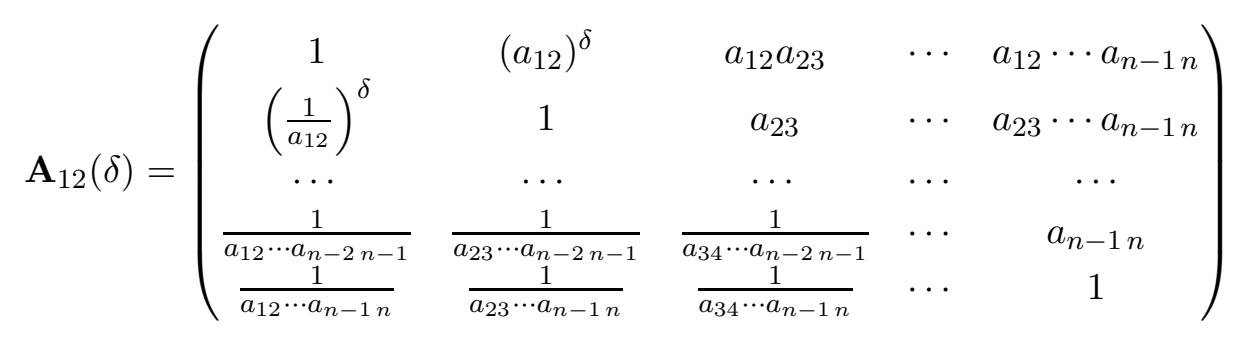


In fact, as index $H C I$ satisfies $\mathrm{A} 2$, by choosing $a_{12}$ we do not lose generality. To prove that $H C I$ satisfies A4 we shall show that

$$
\begin{aligned}
& \frac{\partial H C I\left(\left(\mathbf{A}_{12}(\delta)\right)\right.}{\partial \delta}<0 \text { for } \delta<1 \\
& \frac{\partial H C I\left(\left(\mathbf{A}_{12}(\delta)\right)\right.}{\partial \delta}>0 \text { for } \delta>1
\end{aligned}
$$

It is sufficient to prove it for $H M\left(\mathbf{A}_{12}(\delta)\right)$, since $H C I\left(\mathbf{A}_{12}(\delta)\right)$ is just one of its monotone increasing affine transforms. For notational convenience, let us define $b_{p q}=\prod_{i=p}^{q-1} a_{i i+1}$, e.g. $b_{14}=a_{12} a_{23} a_{34}$. Thus, we can rewrite $H M\left(\mathbf{A}_{12}(\delta)\right)$ in the following way, to keep explicit $\left(a_{12}\right)^{\delta}$ and $\left(1 / a_{12}\right)^{\delta}$

$$
H M\left(\mathbf{A}_{12}(\delta)\right)=\frac{1}{\underbrace{\frac{1+\left(\frac{1}{a_{12}}\right)^{\delta}+\sum_{j=3}^{n} \frac{1}{b_{1 j}}}{1+\underbrace{}_{s_{2}}}}_{\frac{1}{s_{1}}}+\underbrace{\underbrace{}_{i=3} \frac{1}{s_{i}}}_{\frac{1}{\left(a_{12}\right)^{\delta}+1+\sum_{j=3}^{n} \frac{1}{b_{2 j}}}}} .
$$

Considering that only the first two arguments of the sum at the denominator of (42) contain $\delta$, its derivative w.r.t. $\delta$ is

$$
\frac{\partial H M\left(\mathbf{A}_{12}(\delta)\right)}{\partial \delta}=-\frac{n\left[\frac{a_{12}^{-\delta} \ln \left(a_{12}\right)}{\left(1+\left(\frac{1}{a_{12}}\right)^{\delta}+\sum_{j=3}^{n} \frac{1}{b_{1 j}}\right)^{2}}-\frac{a_{12}^{\delta} \ln \left(a_{12}\right)}{\left(\left(a_{12}\right)^{\delta}+1+\sum_{j=3}^{n} \frac{1}{b_{2 j}}\right)^{2}}\right]}{(\cdot)^{2}}
$$

We first consider the case $\delta>1$ and $a_{12}>1$, which implies $\ln \left(a_{12}\right)>0$. Condition (41) is satisfied if in (43) the quantity between square brackets is negative, i.e.

$$
\frac{a_{12}^{-\delta}}{\left(1+\left(\frac{1}{a_{12}}\right)^{\delta}+\sum_{j=3}^{n} \frac{1}{b_{1 j}}\right)^{2}}<\frac{a_{12}^{\delta}}{\left(\left(a_{12}\right)^{\delta}+1+\sum_{j=3}^{n} \frac{1}{b_{2 j}}\right)^{2}} .
$$

With some computation one obtains

$$
\begin{gathered}
\frac{a_{12}^{-\delta}}{\left(\frac{b_{1 n}+a_{12}^{1-\delta} b_{2 n}+\sum_{j=3}^{n-1} b_{j n}+1}{b_{1 n}}\right)^{2}}<\frac{\frac{a_{12}^{\delta}}{a_{12}^{2}}}{\left(\frac{a_{12}^{\delta} b_{2 n}+\sum_{j=2}^{n-1} b_{j n}+1}{b_{1 n}}\right)^{2}} \\
\frac{1}{\left(b_{1 n}+a_{12}^{1-\delta} b_{2 n}+\sum_{j=3}^{n-1} b_{j n}+1\right)^{2}}<\frac{a_{12}^{2 \delta-2}}{\left(a_{12}^{\delta} b_{2 n}+\sum_{j=2}^{n-1} b_{j n}+1\right)^{2}} \\
b_{1 n}+a_{12}^{1-\delta} b_{2 n}+\sum_{j=3}^{n-1} b_{j n}+1>\frac{a_{12}^{\delta} b_{2 n}+\sum_{j=2}^{n-1} b_{j n}+1}{a_{12}^{\delta-1}} .
\end{gathered}
$$

By multiplying both sides times $a_{12}^{\delta-1}$ and given that $b_{1 n}=a_{12} b_{2 n}$, it is

$$
b_{2 n}+a_{12}^{\delta-1} \sum_{j=3}^{n-1} b_{j n}+a_{12}^{\delta-1}>\sum_{j=2}^{n-1} b_{j n}+1 .
$$


Given that $\left(\sum_{j=2}^{n-1} b_{j n}\right)-b_{2 n}=\sum_{j=3}^{n-1} b_{j n}$, then

$$
a_{12}^{\delta-1}\left(\sum_{j=3}^{n-1} b_{j n}+1\right)>\left(\sum_{j=3}^{n-1} b_{j n}+1\right) \Rightarrow a_{12}^{\delta-1}>1 .
$$

Thus, if $a_{12}>1$ and $\delta>1$, one obtains that $\frac{\partial H M\left(\left(\mathbf{A}_{12}(\delta)\right)\right.}{\partial \delta}>0$ and $\frac{\partial H C I\left(\left(\mathbf{A}_{12}(\delta)\right)\right.}{\partial \delta}>0$. If, instead, $0<a_{12}<1$, one knows that $\ln \left(a_{12}\right)<0$, and all inequalities, starting from (44), are inverted. Consequently, $a_{12}^{\delta-1}<1$, which is true for $\delta>1$, and so, also in this case, $\frac{\partial H C I\left(\left(\mathbf{A}_{12}(\delta)\right)\right.}{\partial \delta}>0$. For $\delta<1$, the opposite happens: condition (40) is satisfied and $\frac{\partial H C I\left(\left(\mathbf{A}_{12}(\delta)\right)\right.}{\partial \delta}<0$. This proves that $H C I$ satisfies (20) $)$.

A5 Since pairwise comparison matrices are positive matrices, it follows that $s_{j}>0 \forall j$. Therefore, $H C I(\mathbf{A})$ is a continuous function.

Proof of Proposition 8. As in the other proofs, we shall prove the axioms in sequence.

A1 A2 The proof that $G W$ satisfies axioms $\mathrm{A} 1$ and $\mathrm{A} 2$ is straightforward.

A3 Analogously to Proposition 7 , we shall prove that $G W$ does not satisfy A3 for a whole set of pairwise comparison matrices, instead of providing a single counterexample. Consider the set of inconsistent pairwise comparison matrices $\mathbf{A}$ such that all the greatest elements of each column lie on the same row, i.e. $\exists i^{*}, a_{i^{*} j}>a_{i j} \forall i \neq i^{*}, \forall j$. It is $G W(\mathbf{A})>0$ for all these matrices, since they are inconsistent. Without loss of generality we assume $i^{*}=1$; then, applying $f$ to $\mathbf{A}$ obtaining $f(\mathbf{A})=\mathbf{A}(b)=\left(a_{i j}^{b}\right)_{n \times n}$, when the exponent $b$ tends to $+\infty,(b \rightarrow+\infty)$, it is

$$
\overline{\mathbf{A}(b)} \rightarrow\left(\begin{array}{cccc}
1 & 1 & \ldots & 1 \\
0 & 0 & \ldots & 0 \\
\ldots & \ldots & \ldots & \ldots \\
0 & 0 & \ldots & 0
\end{array}\right) \quad \overline{\mathrm{g}} \rightarrow\left(\begin{array}{c}
1 \\
0 \\
\vdots \\
0
\end{array}\right)
$$

where $\overline{\mathbf{g}}$ is the normalized weight vector obtained from $\overline{\mathbf{A}(b)}$ by means of the geometric mean method. Hence index $G W$ tends to $0,(G W(\mathbf{A}(b)) \rightarrow 0)$ and violates (19).

A5 Index $G W$ is continuous, as it is a sum of continuous functions. 